\title{
TRATAMENTO CIRÚRGICO DAS HÉRNIAS INCISIONAIS: EXPERIÊNCIA PESSOAL USANDO A MALHA DE POLIPROPILENO MONOFILAMENTAR-MÁRLEX
}

\author{
SURGICAL TREATMENT OF INCISIONAL HERNIAS: PERSONAL EXPERIENCE \\ WITH POLYPROPYLENE MONOFILAMENT MESH- MARLEX
}

José Moreira Lima, TCBC ${ }^{1}$

\begin{abstract}
RESUMO: Objetivo: Relatar a experiência pessoal no tratamento cirúrgico de hérnias incisionais com a utilização da malha de polipropileno monofilamentar-márlex. Métodos: Foram operados e seguidos 74 pacientes portadores de hérnia incisional originada de tratamento cirúrgico de diferentes afecções da cavidde abdominal, com idade entre 30 e 94 anos, sendo 39 do sexo feminino e 35 do sexo masculino. Destes 41,9\% foram submetidos a hernioplastia incisional pela primeira vez e $58,1 \%$ já haviam tentado o tratamento sem sucesso. A técnica operatória usada foi a dissecção do saco herniário, ressecção da fibrose resultante de operações anteriores e fixação de tela de márlex substituindo ou reforçando a fáscia transversal por baixo dos músculos da parede abdominal. Resultado: 74 pacientes foram operados e seguidos de 1975 a 1995 . Na última revisão, em maio de 1998, três pacientes haviam falecido, dois de doenças cardíacas e um de doença neoplasia. Apenas um paciente $(1,3 \%)$ apresentou recidiva, no início da experiência. Este foi reoperado e terve sua hérnia incisional curada. Conclusão: o uso da tela de márlex tecnicamente aplicada é o método ideal para a cura definitiva das hérnias incisionais.
\end{abstract}

Descritores: Hérnia incisional, eventração incisional, tela de márlex, próteses.

\section{INTRODUÇÃO}

Os avanços da medicina e das técnicas cirúrgicas cresceram juntos desde o início do século XX. As cirurgias sobre o abdome desenvolveram-se com muita rapidez. Quase todos os cirurgiões, desde o final do século XIX, passaram a praticar intervenções cirúrgicas tanto para correção de lesões naturais da parede abdominal como para tratar doenças da cavidade abdominal.

No início do século XX, não se conhecia bem, ainda, a anatomia e fisiologia da parede abdominal, o que propiciou algumas iatrogenias, independente da competência dos cirurgiões, por ocasião das laparotomias destinadas a tratar várias enfermidades da cavidade abdominal. Mesmo obtendo sucesso no objetivo da intervenção cirúrgica, um problema era eventualmente detectado: a hérnia incisional. Uma de suas causas é a inadequação da sutura do plano anatômico que sustenta a integridade da parede abdominal, que é a fáscia transversal. Este plano, em alguns casos, confunde o cirurgião pouco experiente porque, quando incisada a fáscia geralmente se retrai um pouco, deixando a borda peritoneal exuberante, podendo ficar, no momento da sutura, um pequeno segmento fascial retraído sem envolvimento na sutura. Qualquer solução de continuidade da fáscia transversal permitirá a passagem do peritônio através desta falha, promovendo, assim, a hérnia incisional.

\section{Professor Adjunto Livre-Docente do Departamento de Cirurgia da Faculdade de Medicina da Universidade Federal do} Ceará.

Recebido em 08/12/2000.

Aceito para publicação em 11/12/2001.

Resumo de tese apresentado ao Departamento de Cirurgia como requisito parcial do Concurso de Títulos e Provas para habilitação à Livre-Docência em Clínica Cirúrgica. 


\section{MÉTODO}

Foram estudados 74 pacientes portadores de hérnias incisionais nos Hospitais São Raimundo e São Mateus, sendo 39 do sexo feminino e 35 do sexo masculino com idades entre 30 e 94 anos. Cerca de $62 \%$ se encontravam na faixa etária de 41 a 70 anos e $78 \%$ das lesões foram constatadas na linha mediana. As operações que originaram as hérnias incisionais estão na Tabela 1.

Tabela 1

Operação que originou a hérnia incisional em 74 pacientes

\begin{tabular}{l|c|c}
\hline Operação Original & $\begin{array}{c}\text { No de } \\
\text { Pacientes }\end{array}$ & $\%$ \\
\hline Histerectomia & 17 & 22,8 \\
Cesariana & 11 & 14,9 \\
Apendicetomia & 13 & 17,6 \\
Hernioplastia umbilical & 2 & 2,7 \\
Colecistectomia & 9 & 12,2 \\
Abdome agudo por várias causas & 7 & 9,5 \\
Cirurgia da úlcera péptica & 6 & 8,1 \\
Cirurgia para prostactetomia & 6 & 8,1 \\
Coletomia com colostomia & 2 & 2,7 \\
Esternotomia para cirurgia cardíaca & 1 & 1,3 \\
\hline
\end{tabular}

Alguns pacientes foram submetidos a várias tentativas de cura de sua hérnia incisional, o que pode ser visto na Tabela 2 .

Tabela 2

Índices de reoperações para tratamento da hérnia incisional

\begin{tabular}{lcc}
\hline Reoperação & No de Pacientes & $\%$ \\
\hline 1ª Operação & 31 & 41,9 \\
2ª Reoperação & 26 & 35,1 \\
3- Reoperação & 8 & 10,8 \\
4- Reoperação & 4 & 5,4 \\
5- Reoperação & 3 & 4,1 \\
6- Reoperação & 2 & 2,7 \\
\hline Total & 74 & 100,0 \\
\hline
\end{tabular}

A técnica operatória usada foi a dissecção e fechamento do saco herniário, identificação das bordas sadias da fáscia transversal e colocação de retalho de polipropileno monofilamentar-márlex fixado com fio de prolene 3.0 em pontos separados. A prótese é colocada abaixo dos músculos da parede abdominal mas não deve ficar em contato com as alças intestinais para evitar aderências e fístulas. Também deve ser suficiente para fechar completamente a área comprometida (Figura 1). A aponeurose externa dos músculos da parede abdominal é suturada por cima da pró- tese como pode ser visto na Figura 2. A necessidade de se realizar grandes dissecções impõe a colocação de drenagem eficiente.

Nas grandes eventrações infra-umbilicais adotamos também a dermolipectomia estética e reparadora.

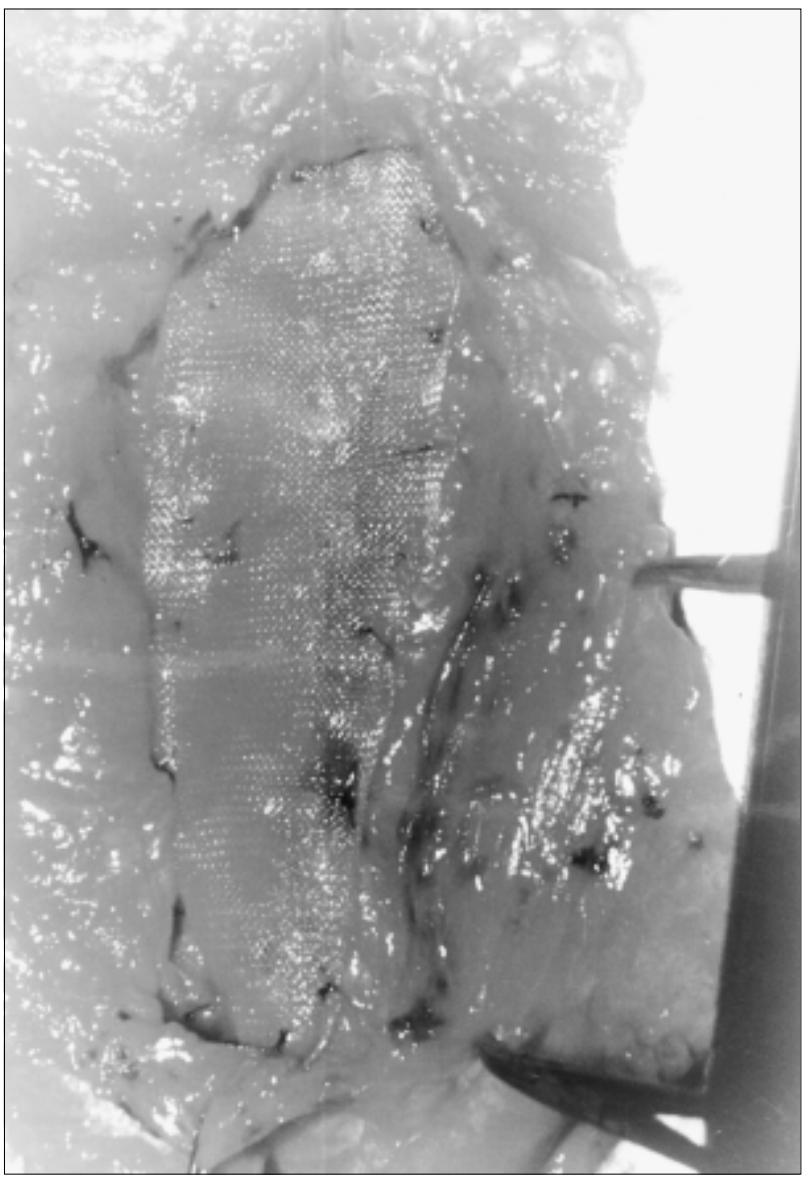

Figura 1 - Prótese de márlex colocada sobre a fáscia

\section{RESULTADOS}

Dos 74 pacientes operados e seguidos no período de 1975 a 1995 houve apenas uma recidiva no início da experiência. Este paciente foi reoperado e teve a sua hérnia incisional curada. Na última revisão, em 1998, apenas um paciente não foi encontrado e três pacientes haviam falecido de outras doenças.

As complicações observadas no pós-operatório dos 74 pacientes referidos são vistas na Tabela 3 .

\section{DISCUSSÃO}

O tratamento cirúrgico das hérnias incisionais vem constituindo motivo de grandes discussões entre os cirurgiões. Trata-se de defeito adquirido em tratamento cirúrgico de várias afecções abdominais que exige reparo eficien- 


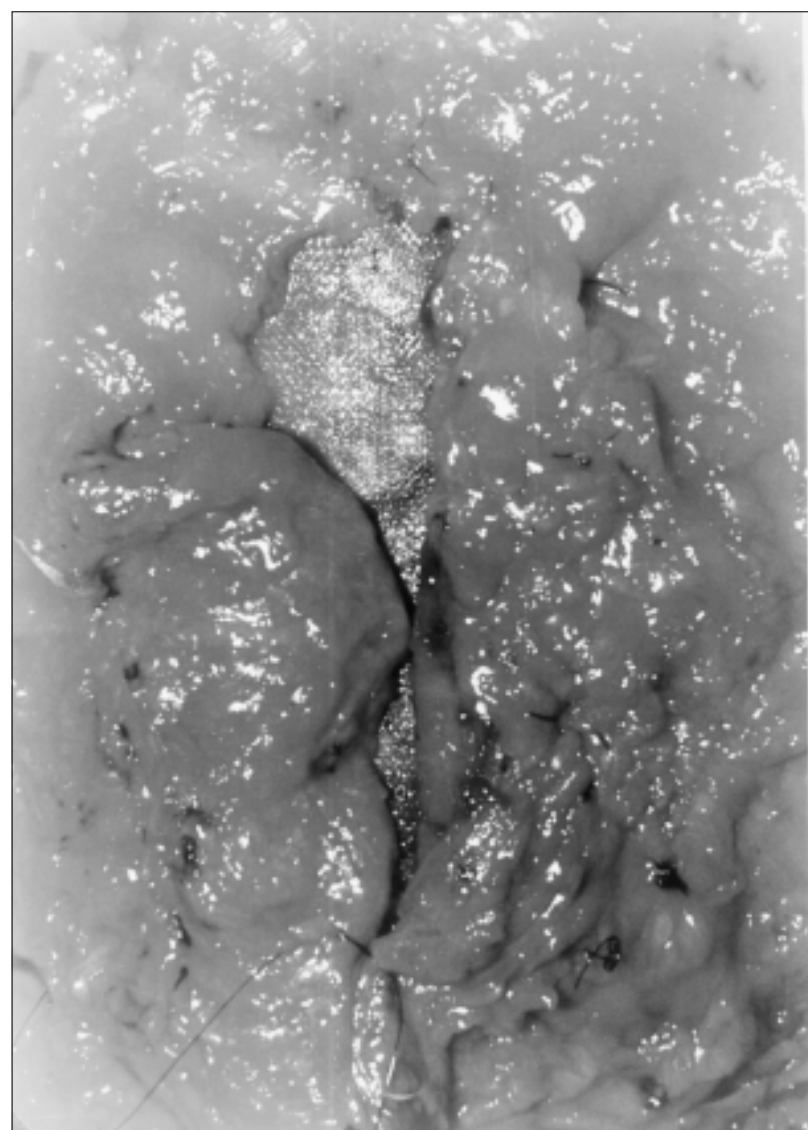

Figura 2 - Fechamento da aponeurose dos retos sobre a prótese de márlex.

Tabela 3

Complicações pós-operatórias observadas em 74 pacientes operados de hérnia incisional

\begin{tabular}{l|c|c}
\hline Complicação & $\begin{array}{c}\text { No de } \\
\text { Pacientes }\end{array}$ & $\%$ \\
\hline Edema na ferida cirúrgica & 3 & 4,1 \\
Infecção da ferida operatória & 2 & 2,7 \\
Formação de seroma após retirar o dreno & 1 & 1,4 \\
Hematoma persistente & 1 & 1,4 \\
Sem complicação & 67 & 90,5 \\
\hline
\end{tabular}

te e definitivo. Muitos são os profissionais que, desde o início do século XX, têm-se preocupado em conseguir solução para este problema. Certamente, o melhor caminho é tentar evitá-lo, dispensando atenção redobrada no fechamento das laparotomias. Mas nem sempre é possível, porque quando há infecção da ferida operatória a parede abdominal torna-se vulnerável. A operação de uma hérnia incisional apresenta sempre maior grau de dificuldade do que a operação original, devendo ser realizada por cirurgião experiente que dedique total atenção para evitar recidivas. A variação dos resultados no tratamento cirúrgico das hérnias incisionais, na literatura, merece ser analisada com profundidade. No início do século XX, Cattell ideali- zou uma técnica com incisão lateral na bainha do músculo reto do abdome, visando utilizar os retalhos para cobrir a falha da fáscia transversal desgastada. O procedimento, entretanto, não apresentou resultados satisfatórios, pois as recidivas se tornaram freqüentes ${ }^{1}$. Identicamente, Mayo propôs uma técnica para tratamento das hérnias umbilicais que consistia no imbricamento das bordas da lesão em sutura separada. O método passou a ser usado por ele e por outros cirurgiões na tentativa de corrigir hérnias incisionais e novamente os resultados não foram satisfatórios: a recorrência continuou alta, variando de $1 \%$ a $50 \%^{2}$. Na Europa, quando há referência ao tratamento convencional das hérnias incisionais estima-se recidiva de $20 \%$ a $40 \%{ }^{3}$. Na Índia, também registra-se recidiva em torno de $40 \%$ nas hernioplastias incisionais convencionais ${ }^{4}$. No Japão, o insucesso no tratamento das hérnias incisionais pelo método convencional incentivou os cirurgiões para o uso de próteses $^{5}$. Na Alemanha, admite-se que cerca de $10 \%$ dos pacientes laparotomizados venham a apresentar hérnia incisional. O reparo dessas hérnias pela técnica de Mayo apresenta recidiva de até $53 \%{ }^{6}$. Nos Estados Unidos registra-se uma grande variedade de resultados. A referência sobre o método convencional, em hérnias incisionais grandes cujo orifício herniário é maior que $10 \mathrm{~cm}$, pode apresentar recidiva em torno de 50\% ${ }^{7}$. Kozoll e McVay registraram incidência de hérnia incisional em 5,7\% das laparotomias praticadas em hospital privado e $11,1 \% \mathrm{em}$ hospital público. Calcularam que na reoperação das hérnias incisionais já recidivadas a recorrência é três a quatro vezes maior ${ }^{8}$. Com índice de recidiva elevado no tratamento das hérnias incisionais, surgiram discussões em busca de alternativas.

Começou o estudo das próteses. Em 1913, em 100 casos de hernioplastia incisional descrita por Loewe, usando o retalho de derme, o resultado foi lentamente se tornando ilusório ${ }^{1}$. Os cirurgiões procuravam um material que preenchesse as características ideais, tais como: resistência, não determinasse reação inflamatória capaz de propiciar infecção e proporcionasse integração aos tecidos vizinhos, formando uma estrutura capaz de substituir a fáscia transversal. Assim, foram utilizados retalhos de pele total, aço inoxidável, tântalo, náilon, mersilene, dácron, téflon, dura-máter humana, entre outros, cada um mostrando suas desvantagens peculiares ao longo do tempo, o que desestimulou os cirurgiões na persistência de seus méto$\operatorname{dos}^{9,26}$. Com a descoberta da malha de polipropileno monofilamentar, passou-se a confiar nesse novo material de prótese $^{10}$, que a partir de 1970 tornou-se o material ideal para reparo de lesões difíceis da parede abdominal.

A malha de márlex se integra muito bem aos tecidos de tal forma que com o passar do tempo se transforma em uma verdadeira parede. Em 1969, dez anos depois da publicação do trabalho de Usher, foi publicado o primeiro trabalho no Brasil, por Falci, enaltecendo a malha de márlex. Ele apresentou o resultado de 100 casos de hérnias operadas com índice de recidiva muito baixo ${ }^{12}$. Embora a divulgação tenha sido lenta, Usher concretizou sua experiência em $1996^{19}$. Desde 1970 Lázaro da Silva experimenta a utilização do saco herniário em vários planos de sutura, 
na tentativa de fechar a falha da parede abdominal ${ }^{13}$. Na experiência dele o resultado é positivo. Atualmente, a literatura está repleta de trabalhos de cirurgiões que, desiludidos com outros métodos, passaram a usar a malha de márlex. O importante é a aplicação técnica da malha. Ela deve ser suficiente para cobrir, com sobra, a lesão da parede abdominal ${ }^{14}$. Pan Chacon obteve resultado negativo de $12 \%$ em 138 hérnias incisionais operadas, mas a malha era colocada por cima do reto abdominal ${ }^{15}$. Na Alemanha, trabalhos recentemente publicados ${ }^{2,5,18}$ referem-se às altas taxas de recidiva nas hernioplastias incisionais, em torno de $40 \%$ a $53 \%$, e enfatizam o uso da malha de polipropileno como solução para resolver esse problema ${ }^{5,6,16}$. Trabalho indiano se refere à formação de sanduíche, isto é, a malha de polipropileno é colocada entre os músculos e o peritônio ${ }^{4}$.

Em torno de 2.000.000 de laparotomias realizadas nos EUA anualmente, cerca de $2 \%$ a $11 \%$ dão origem à hérnia incisional. Após o primeiro reparo, cerca de $30 \%$ a $50 \%$ apresentam insucesso ${ }^{19}$. Com a utilização das próteses, mesmo havendo algumas complicações, o índice de recidiva caiu vertiginosamente. Fazendo comparação entre os materiais de próteses e um novo material derivado do silicone, o TMS-2, ainda em experiência, parece também apresentar excelentes características ${ }^{18}$. Pacientes que apresentam doenças associadas, como disfunção pulmonar, hipertensão, diabetes e obesidade têm maior risco de complicações. O mais importante é a colocação da malha sobre o peritônio, ou seja, abaixo dos músculos da parede abdominal. A malha não deve ficar em contato com as vísceras intra-abdominais, porque promove aderências que podem levar a obstrução intestinal e até mesmo fístulas. Se colocada por cima dos músculos não proporciona segurança à parede abdominal e fica exposta à tela subcutânea, portanto mais vulnerável ao envolvimento de processos infecciosos. A antibioticoterapia e a utilização de drenagem das feridas operatórias não mostraram alterações nos resultados ${ }^{19,21}$.

Com o advento da cirurgia videolaparoscópica, alguns cirurgiões passaram a praticá-la no tratamento das hérnias incisionais ${ }^{20}$. Como o método fica limitado a um pequeno número de cirurgiões, em vista de suas peculiaridades, é provável que não se confirme como tratamento básico para as hérnias incisionais. É certo, porém, que a malha de polipropileno monofilamentar está sendo preferida por todos os interessados em resolver os problemas das hérnias incisionais. A comparação dos resultados nas Tabelas 4 e 5, deste estudo, permite verificar a diferença.

A malha de polipropileno monofilamentar, márlex substituindo ou reforçando a fáscia transversal, de acordo com a descrição técnica deste estudo, é o método eficaz para tratar as hérnias inciosionais proporcionando resultados excelentes.

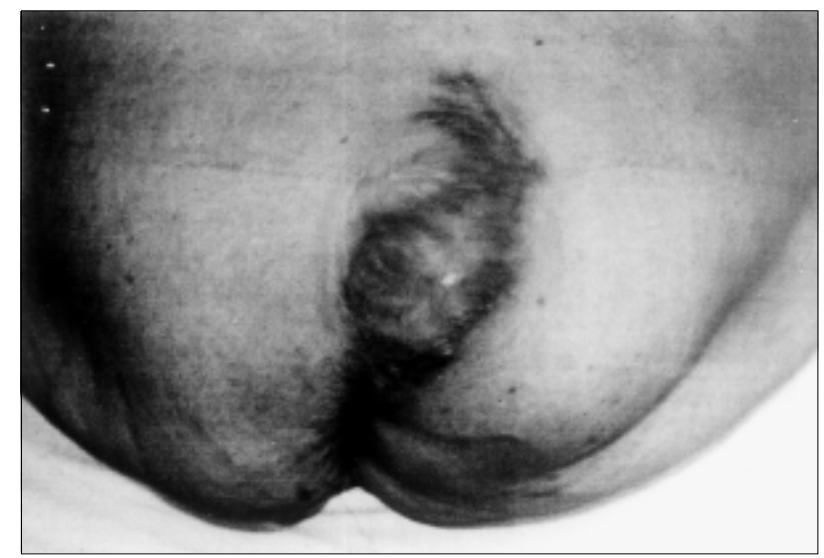

Figura 3 - Volumosa eventração incisional decorrente de operação cesariana na quarta tentativa de cura.

Tabela 4

Índice de recidiva em hernioplastia incisional sem prótese, por vários autores

\begin{tabular}{|c|c|c|c|}
\hline Autor & Ano & $\%$ de Insucesso & País \\
\hline Shelly (Ponka) $)^{25}$ & 1940 & 17,8 & EUA \\
\hline Moreira Lima ${ }^{12}$ & 1980 & 6 & Brasil \\
\hline Ponka $^{25}$ & 1980 & 9 & EUA \\
\hline Kather Neto ${ }^{24}$ & 1997 & $5-44$ & Brasil \\
\hline Paul $^{6}$ & 1997 & $30-50$ & Alemanha \\
\hline Trupka $^{5}$ & 1997 & $40-53$ & Alemanha \\
\hline Hofstetter ${ }^{21}$ & 1998 & $30-40$ & EUA \\
\hline Leber $^{17}$ & 1998 & $30-50$ & EUA \\
\hline Turkçapar ${ }^{22}$ & 1998 & $38-46$ & Japão \\
\hline Matapurkar ${ }^{4}$ & 1999 & $31-44$ & Índia \\
\hline
\end{tabular}

Tabela 5

Índice de recidiva em cirurgia de hérnia incisional com uso da malha de polipropileno monofilamentar-márlex segundo diverso autores

\begin{tabular}{|c|c|c|c|}
\hline Autor & Ano & $\%$ de insucesso & País \\
\hline Drainer $^{27}$ & 1973 & 0,0 & EUA \\
\hline Walker ${ }^{28}$ & 1976 & 11,5 & EUA \\
\hline Usher $^{29}$ & 1979 & 0,0 & EUA \\
\hline Pan Chacon ${ }^{15}$ & 1989 & 12,0 & Brasil \\
\hline Araújo $^{23}$ & 1994 & 4,2 & Brasil \\
\hline Paul \& Lefering ${ }^{6}$ & 1997 & 11,7 & Alemanha \\
\hline Westeber ${ }^{16}$ & 1997 & 6,0 & Alemanha \\
\hline Paul \& Korenkov² & 1998 & 5,1 & Alemanha \\
\hline Trukçapar $^{22}$ & 1998 & 2,0 & Japão \\
\hline Presente estudo & 1999 & 1,3 & Brasil \\
\hline
\end{tabular}




\begin{abstract}
Background: Personal experience in the treatment of incisional hernia with the use of polypropylene monofilament mesh-marlex, is discussed. Method: Seventy-four patients, with ages ranging from 30 to 94 years (39 females and 35 males), with hernia secundary to surgical abdominal procedures, were studied. Half $(41,9 \%)$ of these patients were submitted to surgical correction for the first time, however 58,1\% were recurrences. Dissection of hernia sac, excision of old fibrosis and marlex mesh interposition were carried out to reinforce transversalis fascia under the muscles of the abdominal wall. Results: Seventy-four patients were operated uppon from 1975 to 1995. Last follow-up carried out in 1998 disclosed three deaths not related to previous surgery. There was one recurrence $(1,3 \%)$ at the beginning of our experience, which required second intervention. Conclusion: The use of marlex mesh interposition is a reliable method to correct incisional hernia providing adequate technique is utilized for its fixation
\end{abstract}

Key Words: Incisional hernias; Ventral hernias; Marlex mesh; Prosthesis

\section{REFERÊNCIAS}

1. Lex A, Raia AA, E Acquaroni D. Clinica Cirúrgica Alípio Correia Neto, 4. v. 4. ed. p. 94-100, 1988.

2. Paul A, Korenkov S et al. Mayo-duplication for treatment of incisional hernias following conventional laparotomy: results of a retrospective analysis. Zentralbla. Chir., 1998, v.122: p.862-870.

3. Shukla VK et al. Cardiff repair of incisional hernia. A university hospital experience. Eur. Jour. Surg., 1998,v. 164: p. 271-274.

4. Matapurkar BG et al. Regeneration of abdominal wall aponeurosis. New dimension in marlex peritonela sandwich of incisional hernia. Word J. Surg., 1999v. 23: p. 446-451,.

5. Trupka AW et al. Expanded polyetrafluoroethylene parch for the repair of large abdominal wall hernias. Zentralbl. Chir., 1997,v. 122: p. 879-884.

6. Paul A et al. Repair of incisional hernias: Current pratice in germany. Zentralbl. Chir., 1997, v. 122: p. 859-861.

7. Wantz GE et al. Simposium - Hernia incisional: The problem and the cure american college of surgeons, 1999,p: 429-447,.

8. Kozoll DD. Hérnia Incisional. In: Nyhus LM, Harkins NH. Hernia. Philadelphia: Lippincot, 1966. p. 380-384.

9. Moreira Lima J. Silicone no reparo das hérnias recidivadas. Rev. Fac. Med. UFC, 1973,v. 13: p. 13-16.

10. Usher FC et al. Use of marlex mesh in the repair of incisional hernia. The American Surgeon., 1958,p. 969-974.

11. Falci F. Análise crítica das próteses da parede abdominal. In Hérnia da Parede Abdominal. Rio de Janeiro: Atheneu, 1997. p. 141-151.

12. Moreira Lima J. Hérnia Incisional. Ceará Med., 1980, p. 11-13.

13. Silva AL et al. Emprego do saco herniário na correção cirúrgica de hérnia incisional longitudinal. A Folha Med. 1991,v. 103: p. 73-76.

14. Stopa RE. The french operation in a major anterior incisional hernia. In Moder Hernia Repair. 2. ed. 1996. p. 23-44.

15. Pan Chacon $\mathbf{J}$ et al. Hérnias incisionais abdominais: correção com tela de marlex. Rev. Col. Bras. Cir., 1989, v. XVI: n. 3, p.99-102.

16. Vestweber $\mathrm{KH}$ et al. Results of recurrent abdominal wall hernia repair using polyproplylene mesh. Zentralbl.
Chir., 1997,v.122: p. 885-883,.

17. Leber GE et al. Long-term complications associated with prosthetic of incisional hernias. Arch Surg., 1998, v.133: p.378-382.

18. Cnota AN et al. Development of a novel synthetic material to close abdominal wall defects. The Am. Surg., 1998,v. 64: p.415-418.

19. White TJ et al. Factors affecting wound complications in repair of ventral hernias. American Surg, 1998, v. 64, n. 3, p. 276-280.

20. Phillips EH. Critical appraisal of hernia surgery by laparoscopy. Rev. Col. Bras. Cir., 1993,v. XX, p. 33-36.

21. Hofstetter WL et al. New technique for mesh repair of paracolostomy hernia. Diseases of the Colon \& Rectum., 1998 , v. 41, p.1054-1055.

22. Turkçapar AG et al. Repair of midline incisional henrias using polypropylene grafts. The Japonese Journal of. Surgery. 1998,v. 28:p. 59-63.

23. Araújo FQ. Hérnia incisional. J.B.M., 1994, p. 240-244.

24. Kater-Neto JM. Hérnia incisional. In Hérnia da Parede Abdominal. Rio de Janeiro: Atheneu, 1997. p. 81-93.

25. Ponka JZ. Hernias of the abdominal wall. Saunders WB, Philadelphia. 1980. p. 369-396.

26. Moreira Lima J. Tratamento cirúrgico das hérnias inguinais recidivadas. Modificação da técnica de Mc Vay com o uso da malha de polipropileno monofilamentar-márlex. Rev.Col. Bras. Cir. janeiro de 2000,v. 27 n 1, p. 27 a 31.

27. Drainer IK, Reid DK. Reccurence ter ventral herniorraphy using a polyproplylene mesh prosthesis. Journal of the Royal College of Surgeons, 1973,v. 14, n. 785, p. 255-260.

28. Walker PM, Langer B. Marlex mesh for repair of abdominal wall defects. The Canadian Journal of Surgery, 1976,v. 19, p.211-214.

29. Usher F. New technique for repairing incisional hernia with marlex mesh. The American Journal of Surgery 1979, v.138, p. 740-741.

Endereço para correspondência:

Prof. José Moreira Lima

Rua Vicente Leite, 1800 /1000

60170-151 - Fortaleza-Ce

E-mail: moreira-lima@uol.com.br 